\title{
Heating leads to liquid-crystal and crystalline order in a two-temperature active fluid of rods
}

\author{
Jayeeta Chattopadhyay, Sindhana Pannir-Sivajothi $\odot{ }^{*}$ Kaarthik Varma, Sriram Ramaswamy, \\ Chandan Dasgupta, and Prabal K. Maiti ${ }^{\dagger}$ \\ Centre for Condensed Matter Theory, Department of Physics, Indian Institute of Science, Bangalore 560012, India
}

(Received 2 April 2021; revised 27 September 2021; accepted 22 October 2021; published 16 November 2021)

\begin{abstract}
We report phase separation and liquid-crystal ordering induced by scalar activity in a system of soft repulsive spherocylinders (SRSs) of shape anisotropy $L / D=5$ using molecular dynamics (MD) simulations. Activity is introduced by increasing the temperature of half of the SRSs (labeled hot) while maintaining the temperature of the other half constant at a lower value (labeled cold). The difference between the two temperatures scaled by the lower temperature provides a measure of the activity. Starting from different equilibrium initial phases, we find that activity leads to segregation of the hot and cold particles. Activity also drives the cold particles through a phase transition to a more ordered state and the hot particles to a state of less order compared to the initial equilibrium state. The cold components of a homogeneous isotropic structure acquire nematic and, at higher activity, crystalline order. Similarly, the cold zone of a nematic initial state undergoes smectic and crystal ordering above a critical value of activity while the hot component turns isotropic. We find that the hot particles occupy a larger volume and exert an extra kinetic pressure, confining, compressing, and provoking an ordering transition of the cold-particle domains.
\end{abstract}

DOI: 10.1103/PhysRevE.104.054610

\section{INTRODUCTION}

Active matter [1-3] is characterized by broken detailed balance, through the conversion of a sustained supply of free energy into work at the scale of the individual constituents. This intent of this broad definition is to bring living systems in to the fold of condensed-matter physics while emphasizing their nonequilibrium character. The field has advanced dramatically through experiments on scales from micrometers to kilometers highlighting the qualitative difference between active and passive systems with the same spatial symmetries and through theoretical progress uncovering the laws governing order, fluctuations, and coexistence in active systems [4-22]. Simulations of minimal models are a valuable testing ground for theories and continue to present new observational puzzles [11,15,19,23-25].

Heterogeneous activity is natural: Motility, metabolism, or the speed of other key enzymatic processes [26] can vary among the components of a system. Mixtures of motile and nonmotile $[4,23]$, or more generally active and passive, particles are another case of interest. In the simplest cases these situations are well approximated by assigning thermal baths with different temperatures to different subsets of particles [26-29]. The resulting internal heat flows make the system active in a way that is not obviously identical to the usual prescription of a maintained chemical potential difference $[1,2]$.

Ganai et al. [26] showed that a two-temperature picture provided a natural physical origin for chromatin organization in the nucleus and Joanny and co-workers [27-29] showed

\footnotetext{
*Present address: Department of Chemistry and Biochemistry, University of California San Diego, La Jolla, CA 92093, USA.

†maiti@iisc.ac.in
}

analytically how phase separation arose in two-temperature systems. Spontaneous segregation in two-temperature or active-passive mixtures is widely observed in simulations, Brownian soft disks [30], polymers [31,32], and binary Lennard-Jones systems [33] where activity leads to phase separation and formation of crystalline domains. Moreover, in the literature, it has been reported that nonreciprocal interactions yield two different temperatures in dusty plasmas [34,35] and diffusiophoretic colloids [36].

These studies show the emergence of collective behavior uniquely associated with activity even when the structure and dynamics at the particle scale are isotropic. Anisotropy, however, is ubiquitous in the living world in the form of the shape and movement of microorganisms, the long persistence lengths of biopolymers [37,38], and the mesogenic nature of lipids. Liquid-crystalline order [39] was central to the inception of active-matter research [1]. Activity in models of liquid-crystalline order generally enters as a selfpropelling force vector $[1,2,6,23,40-48]$ or an active stress tensor [49-52]; even active isotropic baths as in [53] are created by persistent vector or tensor processes. We explore the statistical mechanics of anisotropic particles driven by a strictly scalar manifestation of activity in a two-temperature system of soft repulsive spherocylinders (SRSs). We ask the following questions.

(i) How does the phase behavior of three-dimensional soft rods depart from its equilibrium form when activity is introduced?

(ii) What distinctive features can be traced specifically to the two-temperature nature of the system, in which activity leads to phase separation and resides not in any one of the particles, but at the interfaces between regions of hot and cold particles?

In this paper, we answer these questions through molecular dynamics simulation (MD) of a collection of SRSs of shape 
anisotropy $L / D=5$ with a purely repulsive interaction. Activity is introduced by connecting half of the particles (labeled "hot") to a thermostat of higher temperature, while the rest of the particles (labeled "cold") remain connected to a thermostat of a lower temperature equal to that of the initial equilibrium system. The difference between the two temperatures scaled by the lower temperature is taken to be a measure of the strength $\chi$ of the activity. We describe the model and the simulation protocol in detail in Sec. II.

Our simulation study demonstrates that unlike spherical colloidal particles, where prominent effects of activity are found only when the strength of the activity is high, for both scalar $(\chi \sim 30)[27,30]$ and vector activity (Péclet number $\mathrm{Pe}>50$ ) [19], a variety of interesting phenomena are observed for colloidal rods in a much smaller range of values of the activity parameter $1<\chi<4$. This observation suggests that the two-temperature model should be an experimentally feasible system for studying the effects of scalar activity in collections of rodlike particles. In this regard, the critical activity $\chi_{c}$, defined as the value of $\chi$ at which macroscopic phase separation starts to occur, shows a nonmonotonic dependence on the packing fraction $\eta$, decreasing with increasing $\eta$ in the liquid regime and increasing again in the crystalline regime. A detailed analysis of the phase separation can be found in Sec. III A.

The segregated zones develop different liquid-crystalline (LC) structures depending on the level of activity and the reference equilibrium phase of the system at zero activity. We observe the cold particles undergoing a phase transition towards a more ordered state and the hot particles towards a less ordered state as compared to the initial equilibrium state. If the system at zero activity is in the isotropic ( $I$ ) fluid phase, the cold domains that emerge are nematic $(N)$ and, at higher activities, crystalline $(K)$, while the hot particles remain in the isotropic phase with reduced density. Similarly, a homogeneous nematic reference configuration shows smectic (Sm) and crystalline cold domains and isotropic structure in the hot domain. As a result, the phase boundary of the $I-N$ transition shifts towards lower density for the cold particles and higher density for the hot particles. Different LC phases are identified by calculating the local nematic order parameter and suitable pair correlation functions. Finally, we analyze interfacial properties and find that LC ordering in the lower packing fractions (starting from $I$ and $N$ phases) is governed by local balance of pressure across the interface: Higher temperature induces higher kinetic pressure in the hot zone, which is compensated in the cold zone by increasing virial pressure. Thus mechanical stability is maintained at the interface. Detailed analyses of the segregated phases and interfacial properties are presented in Secs. III B-III D.

The extraordinary nonequilibrium feature that we wish to highlight is that an enhancement of the temperature of a fraction of the particles gives rise to enhanced LC ordering in the remaining particles at any packing fraction.

\section{MODEL AND SIMULATION DETAILS}

We model the system as a collection of SRSs (cylinders with hemispherical caps). The shape anisotropy $A$ is defined by the ratio of length $L$ and diameter $D, A=$

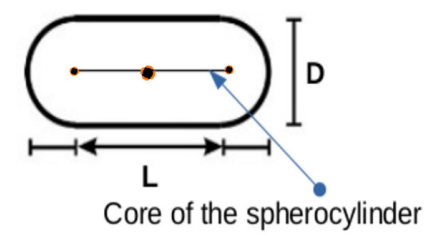

(a)

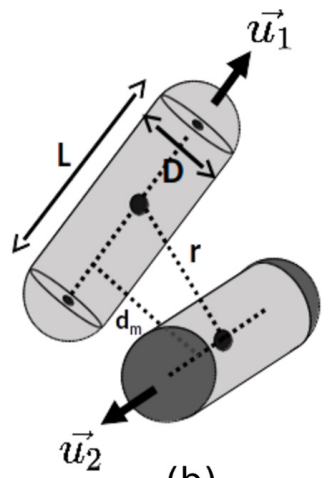

(b)
FIG. 1. Schematic diagram of SRSs. (a) The line segment joining the centers of the two hemispheres is known as core of the spherocylinder. (b) Here $\vec{u}_{1}$ and $\vec{u}_{2}$ denote the orientations of spherocylinders 1 and 2, respectively, $r$ is the distance between their centers of masses, and $d_{m}$ is the shortest distance that determines the interaction potential between them. Panel (b) is adapted from Ref. [67].

$L / D$ (Fig. 1). Spherocylinders interact through the WeeksChandler-Andersen potential (WCA) [54] generalized to nonspherical bodies [55]

$$
U_{S R S}= \begin{cases}4 \varepsilon\left[\left(\frac{D}{d_{m}}\right)^{12}-\left(\frac{D}{d_{m}}\right)^{6}\right]+\epsilon & \text { if } d_{m}<2^{1 / 6} D \\ 0 & \text { if } d_{m} \geqslant 2^{1 / 6} D,\end{cases}
$$

where $d_{m}$ is the shortest distance between two spherocylinders that determines their relative orientation and interacting force [56-58]. Note that representing spherocylinder by a line of interacting spheres can also be used to study various LC phases [59].

We perform MD simulations in the constant numberpressure-temperature (NPT) ensemble, using a Verlet algorithm [60] to update the positions and velocities of the particles and quaternion-based rigid-body dynamics [61-65] for rotational motion. The temperature and pressure of the system are maintained using a Berendsen thermostat and manostat [66] with a temperature relaxation time $\tau_{T}=0.05$ and pressure relaxation time $\tau_{P}=2.00$, respectively. Thermodynamic and structural quantities are scaled by system parameters (i.e., $\epsilon$ and $D$ ) and calculated in reduced units: temperature $T^{*}=k_{B} T / \epsilon$, pressure $P^{*}=\frac{P v_{\text {hsc }}}{k_{B} T}$, and packing fraction $\eta=v_{\text {hsc }} \rho$, where $\rho=\frac{N}{V}$ and $v_{\text {hsc }}=\pi D^{2}\left(\frac{D}{6}+\frac{L}{4}\right)$ is the volume of the spherocylinder.

We prepare the system initially in a hexagonal-closepacked crystalline structure. As the constituent particles are asymmetrical in shape, we choose the numbers $n_{x}, n_{y}$, and $n_{z}$ of unit cells in the $x, y$, and $z$ directions, respectively, in such a way that the simulation box can be constructed in a nearly cubic geometry. If $n_{u}$ is the number of spherocylinders in one unit cell then the total number of spherocylinders $N=$ $n_{u} \times n_{x} \times n_{y} \times n_{z}$. The usual periodic boundary condition and minimum image condition are used. A system of $N=1024$ is built by choosing $n_{x}=n_{y}=16$ and $n_{z}=4$. The ratios of the dimensions of the simulation box are $L_{x} / L_{y}=1.16$ and $L_{z} / L_{y}=1.68$. Later we increase the system size to $N=4096$ to check for finite-size effects. 


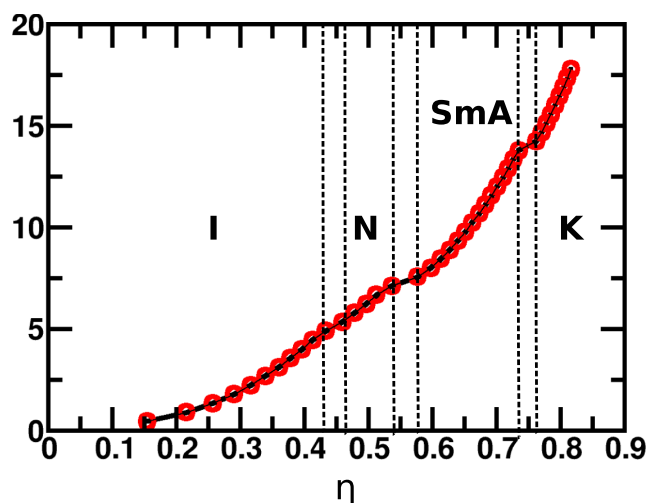

(a)

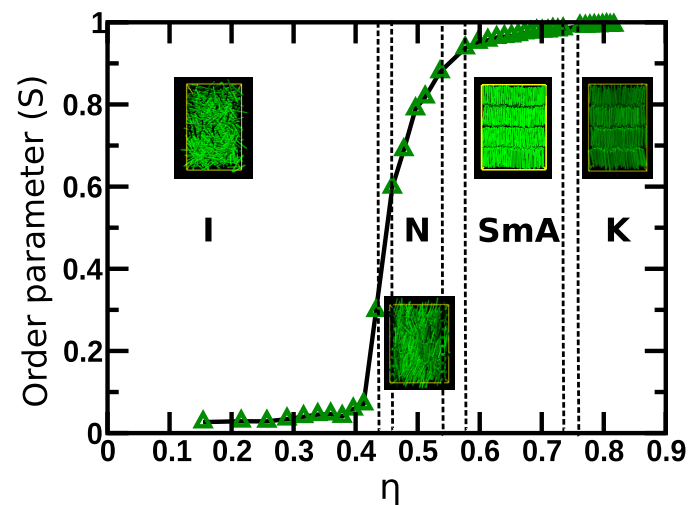

(b)

FIG. 2. (a) Equation of state and (b) nematic order parameter $S$ vs packing fraction $\eta$ for the system of soft repulsive spherocylinders with shape anisotropy $L / D=5.00$. Thermodynamic quantities are defined in reduced units: packing fraction $\eta=\rho v_{\text {hsc }}$ and pressure $P^{*}=P v_{\text {hsc }} / k T$, where $v_{\text {hsc }}$ is the volume of the spherocylinder. Here we observe four stable phases: isotropic $(I)$, nematic $(N)$, smectic $(\mathrm{Sm})$, and crystal $(K)$. The vertical dashed lines indicate coexisting regions near the phase transition points.

After building the system, we equilibrate it at $T^{*}=5.00$. We then establish the equilibrium phase diagram for this temperature by slowly varying the pressure to melt the system. We simulate for a range of pressures $P^{*}$ from 20 to 0.05 which spans the crystal to isotropic phases. The ordering transitions are located by calculating the nematic order parameter and suitable pair correlation functions. The order parameter for the nematic phase is a traceless symmetric tensor $\boldsymbol{Q}$, defined below, which is used to obtain the scalar nematic order parameter $S$, which is the largest eigenvalue of $\boldsymbol{Q}$, and the corresponding eigendirection, which is the director $\mathbf{n}$. A value of $S$ consistent with zero defines the isotropic phase. In highly ordered states, $S \simeq 1$. Let $u_{i}^{\alpha}$ be the $\alpha$ th component of the orientation vector of spherocylinder $i$. Then we define

$$
Q_{\alpha \beta}=\frac{1}{N} \sum_{i=1}^{N}\left(\frac{3}{2} u_{i}^{\alpha} u_{i}^{\beta}-\frac{1}{2} \delta_{\alpha \beta}\right) .
$$

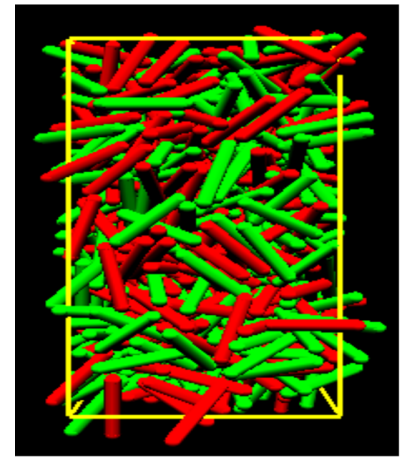

(a)

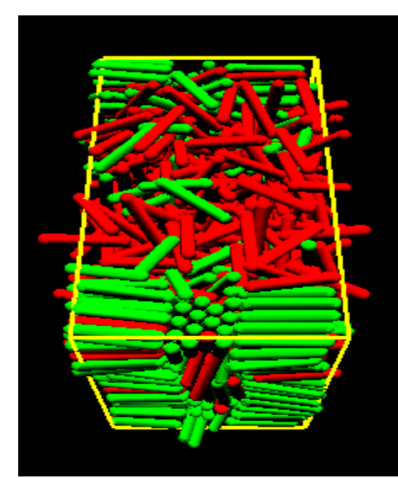

(b)
FIG. 3. (a) Equilibrium configuration of $N=1024$ soft spherocylinders at the state point $\left(\eta=\rho v_{0}=0.36, T^{*}=5.00\right)$ in the absence of activity $\chi=0.00$. Both hot (red) and cold (green) particles are well mixed at the same temperature. (b) Steady-state configuration after phase separation at $\chi=5.00$. It is clearly visible that cold particles are segregated and ordered, whereas the surrounding hot particles are disordered.
We introduce activity by choosing half of the particles randomly and assigning a higher temperature to them while keeping the other particles' temperature fixed at a lower value equal to that of the initial equilibrium system. Let $T_{h}^{*}$ and $T_{c}^{*}$ be the temperatures of the hot and cold particles, respectively. Initially we equilibrate the system at $T_{h}^{*}=T_{c}^{*}=5.00$ and then increase $T_{h}^{*}$ in steps $T_{h}^{*}=5.00 \rightarrow 7.50 \rightarrow 10.00 \rightarrow$ $\cdots \rightarrow 30.00 \rightarrow 50.00$, allowing the system to reach a steady state after each increase in $T_{h}^{*}$, keeping the volume of the simulation box constant throughout the simulation. As a result of heat exchange, the measured values of effective temperatures $T_{h}^{\text {eff }}$ and $T_{c}^{\text {eff }}$ of the two populations, as defined by their steady-state average kinetic energies, differ from those of their thermostats:

$$
T_{h}^{*}>T_{h}^{\mathrm{eff}}>T_{c}^{\mathrm{eff}}>T_{c}^{*} .
$$

We parametrize activity by

$$
\chi=\frac{T_{h}^{*}-T_{c}^{*}}{T_{c}^{*}} .
$$

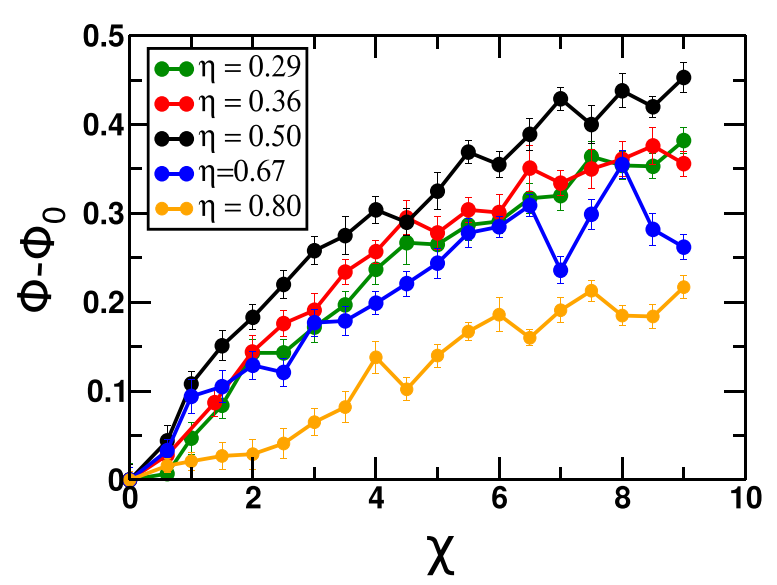

FIG. 4. Density order parameter $\phi$ vs activity $\chi$ at several packing fractions $\eta$ of the system. 


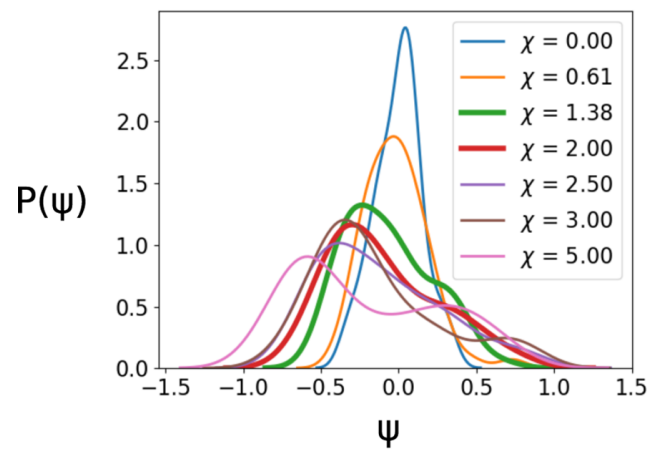

(a)

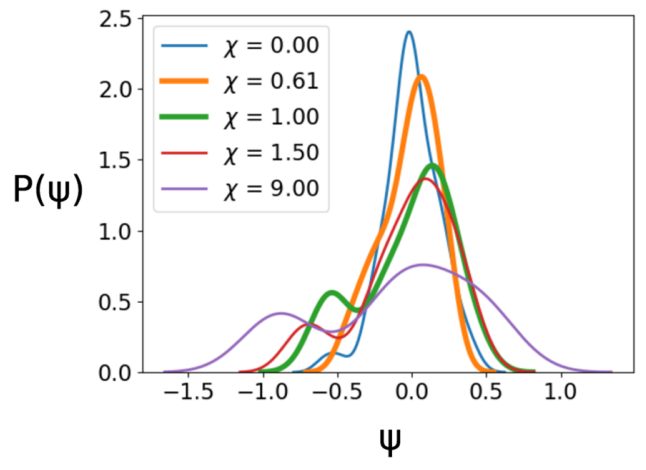

(c)

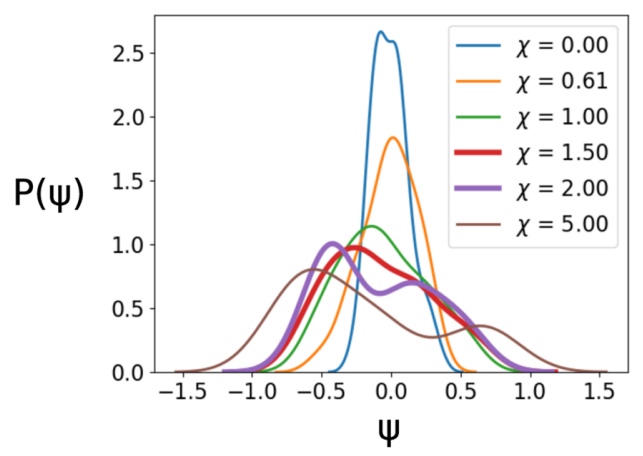

(b)

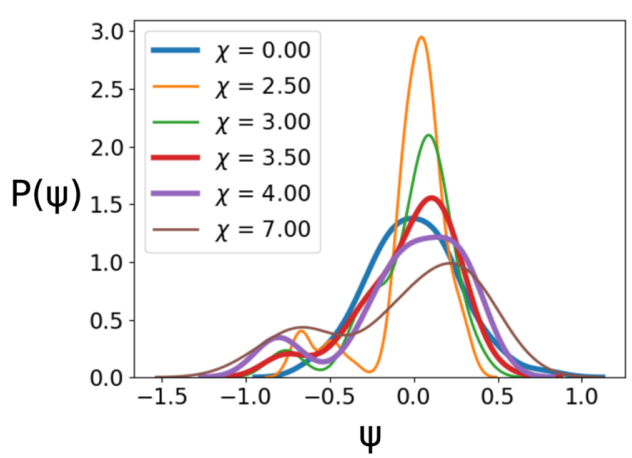

(d)

FIG. 5. Distribution of $\psi, P(\psi)$, at different activities $\chi$ for several different packing fractions $\eta$. Critical activity $\chi_{c}$ is defined as the value of $\chi$ at which $P(\psi)$ develops bimodality. However, we find a plateau region just below the calculated $\chi_{c}$ which is the signature of the emergence of phase separation. Therefore, the exact value of $\chi_{c}$ lies within the following ranges: (a) for $\eta=0.36$, in the initial isotropic phase, $\chi_{c}=1.38-2.0$; (b) for $\eta=0.50$, in the initial nematic phase, $\chi_{c}=1.50-2.00$; (c) for $\eta=0.67$, in the initial smectic phase; $\chi_{c}=0.61-1.00$; and (d) for $\eta=0.80$, in the initial crystal phase, $\chi_{c}=3.50-4.00$. Note that, in the crystal phase, bimodality appears from $\chi=2.50$ to 3.00 . However, we do not consider this the critical activity $\chi_{c}$ as the higher peak arises at $\psi=0$, indicating that most of the particles are mixed. In contrast, at $\chi=3.50-4$, both of the peaks occur at a nonzero value of $\psi(\psi=-0.8,0.25)$. The $P(\psi)$ for the two limits on $\chi_{c}$ are shown as thick lines. The range of $\chi_{c}$ is represented by a gray band in the phase diagram (Fig. 6). The nonzero weight for $|\psi|>0$ arises from the fitting procedure.

For the active case, i.e., for $\chi \neq 0$, we choose the thermostat relaxation time $\tau_{T}=0.01$ for both types of particles. We run the simulation for $3 \times 10^{5}$ to $4 \times 10^{5}$ integration time steps to reach steady state and another $10^{5}$ steps to calculate thermodynamic and structural quantities. We use an integration time step $\delta t=0.001$ in units of the natural timescale $D \sqrt{m / \epsilon}$.

\section{RESULTS AND ANALYSIS}

We present the equilibrium phase diagram of SRSs for $L / D=5$ at $T^{*}=5$ and observe four stable phases: (i) crystal, (ii) smectic $A(\operatorname{Sm} A)$, (iii) nematic, and (iv) isotropic (Fig. 2). The critical values of thermodynamic quantities at phase transition points match well with previous results by Cuetos et al. [67,68].

\section{A. Activity-induced phase separation}

Our system receives a sustained flux of energy which the hot particles draw from the hot bath and transfer through collisions to the cold particles, which in turn reject the excess to the cold bath. At a steady state, the power gained by the hot particles is equal to the power transferred by the cold particles keeping the net energy flux into the system zero. A region occupied predominantly by hot particles

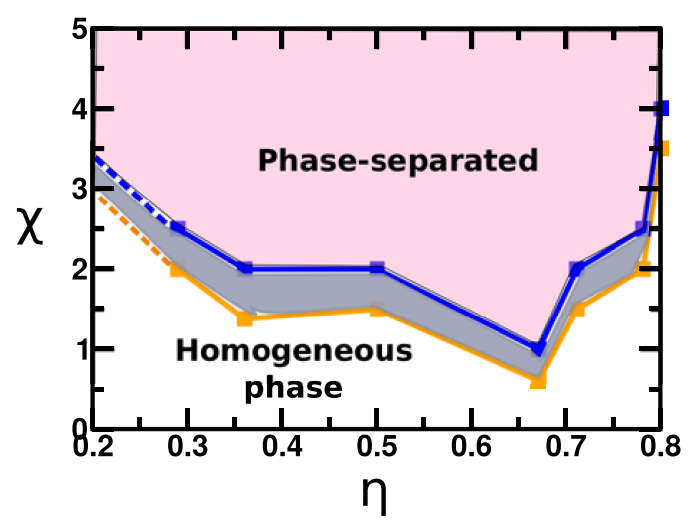

FIG. 6. Phase diagram in the state space $\chi$ vs $\eta$. The pink-shaded area indicates the phase-separated region and the nonshaded area indicates the region of the homogeneous phase where hot and cold particles are well mixed. The blue and orange lines indicate upper and lower limits of critical activity $\chi_{c}$ and the in-between area of gray shade denotes the range of possible values of $\chi_{c}$. Dotted lines are extrapolations from the calculated data. 
(a)

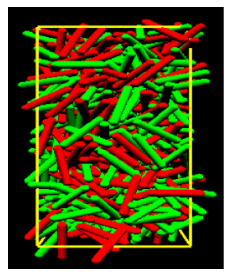

$x=0.00$

$S=0.02-1$ (b)

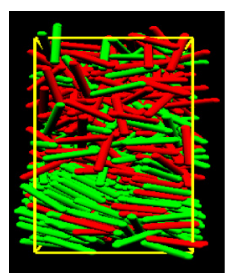

$\chi=2.00$

$\mathrm{S}_{\text {cold }}=0.50-\mathrm{N}$

Shot $=0.07-1$ (c)

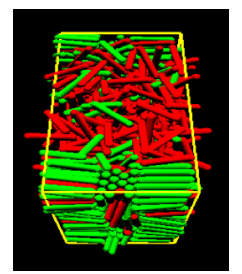

$X=5.00$

$S_{\text {cold }}=0.62$

Domain crystal
Shot $=0.06-1$ (d)

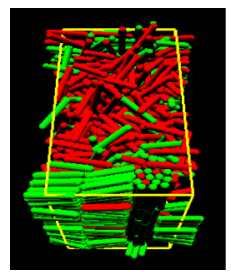

$\chi=9.00$

$\mathrm{S}_{\text {cold }}=0.64$ -

Domain crystal (DK)

Shot $=0.06-1$ (e)

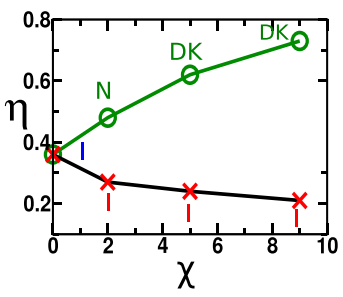

CO $\eta_{\text {cold }}$

FIG. 7. Segregated structures for the initial isotropic phase $(I) \eta=0.36$ : (a) initial configuration in the absence of activity, (b) nematic $(N)$ ordering in the cold cluster, and (c) and (d) multidomain crystal (DK) in the cold cluster. (e) Packing fractions in the segregated zone corresponding to the aforementioned activities. The phases in each zone are mentioned for each activity. Here $S_{\text {cold }}$ and $S_{\text {hot }}$ are the nematic order parameters of cold and hot particles, respectively, and $\eta_{\text {cold }}$ and $\eta_{\text {hot }}$ are the packing fractions in the cold and hot zones, respectively. In the case of (c) and (d), $S_{\text {cold }}$ is much lower than that for usual crystalline ordering as it is calculated by averaging over all the domains with different orientations of the directors. However, the local ordering in each domain is much higher $\left(S_{\text {cold }}^{l o c}=0.90\right)$, which indicates crystalline order. The lines drawn in (e) are a guide to the eye.

tends to expand relative to one with cold particles. This opens up the possibility of phase separation by hot particles self-consistently sequestering a domain of cold particles. As $\chi$ is increased, we indeed see such an effect, locally at first and then macroscopically with a well-defined interface (Fig. 3).

The extent of phase separation is quantified from the spatial distribution of hot and cold particles. To do so, we divide the simulation box into a number of subboxes $N_{\text {box }}$ and for each subbox $i$ we calculate the absolute number difference of hot $\left(n_{h}^{i}\right)$ and cold $\left(n_{c}^{i}\right)$ particles divided by total number of particles in that subbox. This quantity is denoted as the order parameter and is averaged over all the subboxes and also over a sufficiently large number of steady-state configurations as given by

$$
\phi=\frac{1}{N_{\text {box }}}\left\langle\sum_{i=1}^{N_{\text {box }}} \frac{\left|n_{c}^{i}-n_{h}^{i}\right|}{\left(n_{c}^{i}+n_{h}^{i}\right)}\right\rangle_{s s},
$$

where $\langle\cdots\rangle_{s s}$ denotes a steady-state average over a sufficiently large number of configurations. The selection of the number of subboxes is arbitrary; we choose it such that (in our case, $N_{\text {box }}=4^{3}$ ) each box contains enough particles to obtain good statistics. Ideally, in the absence of activity (at $T_{c}^{*}=T_{h}^{*}=$ $5.00), \phi$ should be zero; however, for a finite system size, it can be nonzero; hence we offset it by the initial value $\phi_{0}$, $\phi \rightarrow \phi-\phi_{0}$.

In Fig. 4 we observe that $\phi$ increases monotonically with $\chi$ up to a certain value and then saturates. The reason is that local separation emerges at lower activities and increases until a well-defined interface is formed (see the Appendix and Fig. 18 for a detailed calculation of macroscopic phase separation). The value of $\chi$ at which phase separation starts to occur macroscopically is defined as the critical activity $\chi_{c}$. However, calculating $\chi_{c}$ from Fig. 4 is difficult as the crossover between mixed and phase-separated states is not sharp enough. Hence, we identify $\chi_{c}$ from the following criteria: We define a quantity $\psi$ that signifies the number difference between hot and cold particles in each subbox $\psi=\left\langle\frac{n_{c}-n_{h}}{n_{c}+n_{h}}\right\rangle_{s s}$ and compute the distribution $P(\psi)$ over the subboxes. The activity at which

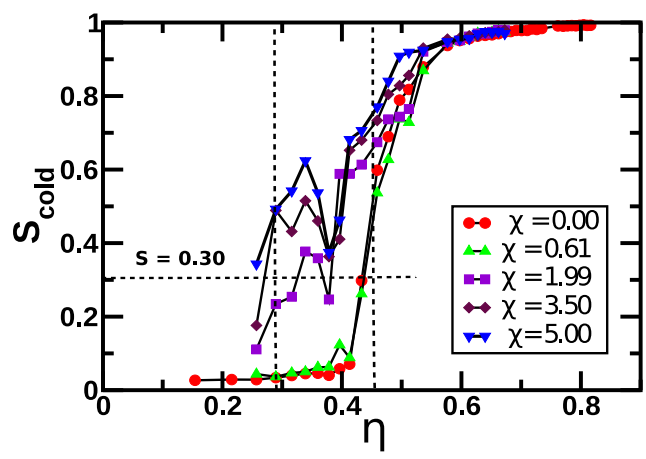

(a)

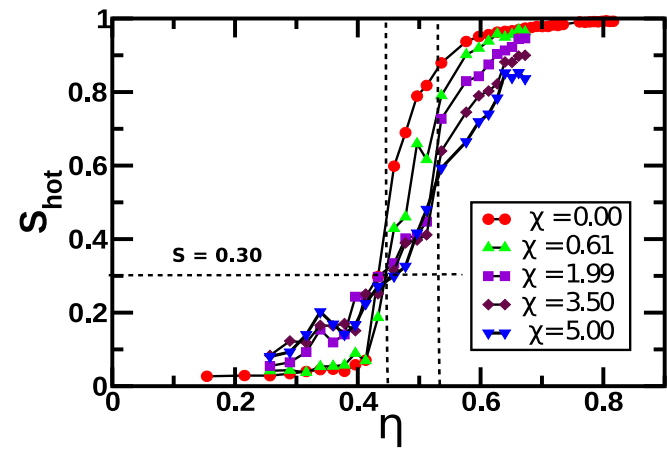

(b)

FIG. 8. Nematic order parameter of (a) cold particles $S_{\text {cold }}$ and (b) hot particles $S_{\text {hot }}$ vs packing fraction $\eta$ at different activities $\chi$. The vertical dashed lines indicate the shift of the $I-N$ phase boundary towards lower packing fraction for cold particles and higher packing fraction for hot particles. The horizontal dashed line indicates the critical value of the order parameter $(S=0.30)$ assumed to indicate the isotropic to nematic transition. 
(a)

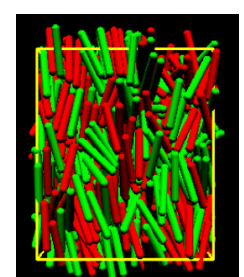

$X=0.00$

$\mathrm{S}=0.75-\mathrm{N}$ (b)

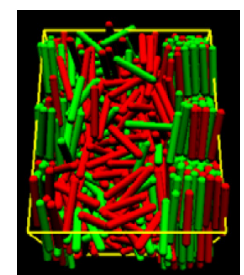

$\chi=2.00$

$\mathrm{S}_{\text {cold }}=0.97-\mathrm{SmA}$

Shot $=0.10-1$ (c)

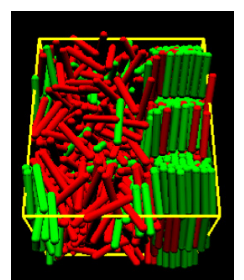

$\chi=5.00$

$\mathrm{S}_{\text {cold }}=0.99-\mathrm{K}$

Shot $=0.10-1$ (d)

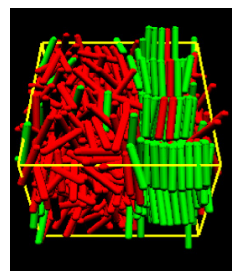

$\chi=9.00$

$\mathrm{S}_{\text {cold }}=0.99-\mathrm{K}$

Shot $=0.08-1$ (e)

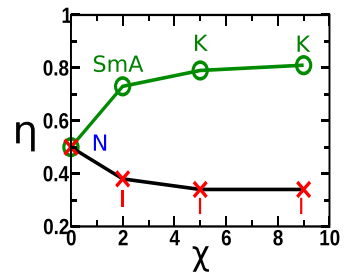

QO ncold

FIG. 9. Segregated structures in a nematic initial configuration $(\eta=0.50)$ : (a) initial structure in the absence of activity, (b) smectic ordering in the cold zone and isotropic structure in the hot zone, and (c) and (d) crystalline ordering in the cold zone and isotropic structure with reduced density in the hot zone. (e) Packing fractions in the segregated zone corresponding to the aforementioned activities. The phases in each zone are labeled for each activity. The parameters are the same as in Fig. 7.

$P(\psi)$ develops bimodality is considered to be the critical activity $\chi_{c}$ of the system.

In Fig. 5 we calculate $\chi_{c}$ from $P(\psi)$ for different packing fractions corresponding to the different initial phases. In the case of lower packing fractions $\eta=0.36$ [Fig. 5(a)], bimodality appears at a higher value than actual $\chi_{c}$. However, we observe a plateau regime with shifted unimodal peak which is the signature of the emergence of phase separation. This is also seen for other packing fractions just below the calculated $\chi_{c}$. Therefore, for each $\eta$, we define a range of $\chi$ within which the exact value of $\chi_{c}$ lies. With these observations, we present a complete phase diagram in the state space $(\chi$ vs $\eta$ ), showing parametric regions of mixed and phase-separated states (Fig. 6).

From Fig. 6 we find that $\chi_{c}$ decreases with the increase of packing fraction $\eta$ up to a value of $\eta=0.67$. This can be due to the fact that the interaction between hot and cold particles is higher for dense systems, which causes fast dissipation of hot particles' energy. Beyond $\eta=0.67$, crystalline order emerges and $\chi_{c}$ increases again as a function of $\eta$ [Fig. 5(d)]. The possible reasons are (i) in an extremely dense system many hot particles are stuck in the cold zone, which requires a larger amount of energy to overcome the barrier, or (ii) the relaxation is very slow in the crystal phase compared to the liquid-crystal phases. Therefore, it may require a longer time to undergo phase separation at lower activity. However, it is interesting to note that critical activity lies in a very small range $1.0<\chi_{c}<4.0$, i.e., a ratio of temperatures $2.0<$ $T_{h}^{\text {eff }} / T_{c}^{\text {eff }}<5.0$ for the entire range of $\eta$. This observation indicates that the two-temperature model should be a reliable system to observe the effect of scalar activity in colloidal rods experimentally.

\section{B. Activity-induced liquid-crystalline ordering}

Hot particles exert an active kinetic pressure along the hot-cold interfaces, which drives an ordering transition in the cold particles. The ordered structures in the phase-separated domains depend on the overall packing fraction $\eta, T^{*}$, and $\chi$. Starting from the state points in the equilibrium $\eta-P^{*}$ phase diagram corresponding to isotropic, nematic, and other phases, we observe the cold domains undergoing phase transitions towards more ordered states and the hot domains towards less ordered states, as compared to the initial equilibrium state. The extent of the segregated zone is quantified by the density profile normal to the interface, which we discuss later (in Sec. III D). Different phases are characterized by calculating the local nematic order parameter $S$ and suitable positional and orientational pair correlation functions.

\section{Initial isotropic configuration}

In Fig. 7 we show the emergence of various phases in the hot and cold regions under different activities, starting from an initial isotropic phase. The critical activity $\chi_{c}$ for (a)

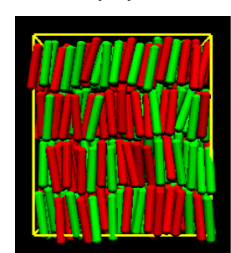

$x=0.00$

$\mathrm{S}=0.97-\mathrm{Sm}$ (b)

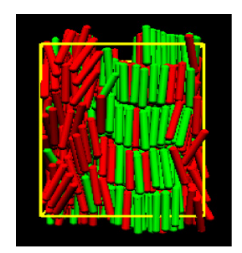

$x=2.00$

$\mathrm{S}_{\text {cold }}=0.99-\mathrm{K}$

Shot $=0.92-\mathrm{N}$ (c)

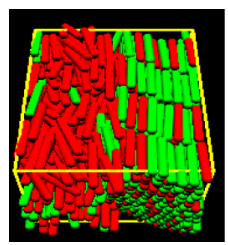

$X=5.00$

$\mathrm{S}_{\text {cold }}=0.99-\mathrm{K}$

Shot $=0.81-\mathrm{N}$ (d)

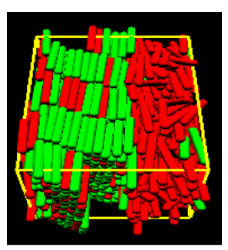

$X=9.00$

$\mathrm{S}_{\text {cold }}=0.99-\mathrm{K}$

Shot $=0.63-\mathrm{N}$ (e)

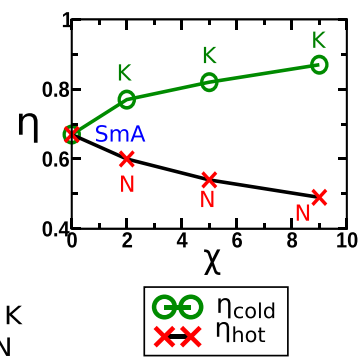

FIG. 10. Segregated structures in a smectic initial phase $(\eta=0.67)$ : (a) initial configuration in the absence of activity and (b)-(d) crystal structures in the cold region and nematic structures in the hot region at different activities. (e) Packing fractions in the segregated zone corresponding to the aforementioned activities. The phases in each zone are labeled for each activity. The parameters are the same as in Fig. 7. 
(a)

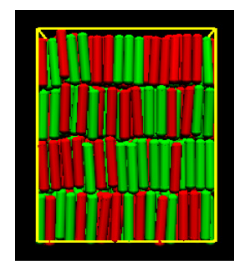

$x=0.00$

$\mathrm{s}=0.99-\mathrm{K}$ (b)

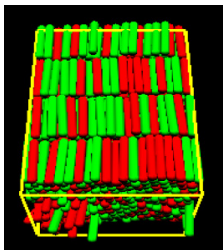

$x=2.00$

$\mathrm{S}=0.99-\mathrm{K}$ (c)

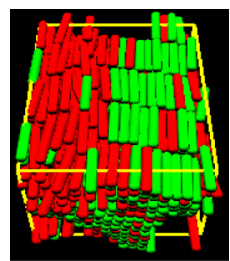

$\chi=5.00$

$\mathrm{Scold}=0.99-\mathrm{K}$ (d)

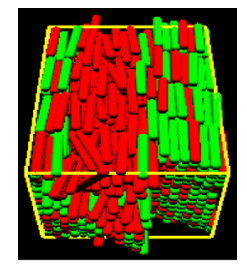

$X=9.00$

$\mathrm{S}_{\text {cold }}=0.99-\mathrm{K}$

Shot $=0.92-\mathrm{N}$ (e)

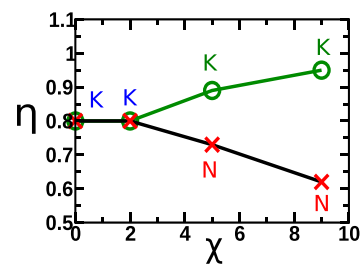

GO $\eta_{\text {cold }}$

$\times$. $\eta_{\text {hot }}$

FIG. 11. Segregated structures in a crystalline initial state $(\eta=0.80)$ : (a) initial configuration in the absence of activity and (b) no phase separation at $\chi=2.00$, with both hot and cold particles in the crystal phase. (c) and (d) Crystalline structure in the cold region with higher packing fraction and nematiclike structure in the hot region. This is far from the usual nematic phase as the breakdown of layering reduces the packing fraction significantly but the nematic order parameter is much higher than that in the equilibrium nematic phase. (e) Packing fractions in the segregated zone corresponding to the aforementioned activities. The phases in each zone are labeled for each activity. The parameters are the same as in Fig. 7.

phase separation lies between 1.38 and 2.00. Cold particles undergo a transition to a nematic phase at lower activities [Fig. 7(b)] which eventually turns into crystalline order at higher activities [Figs. 7(c) and 7(d)]. However, hot particles remain in the isotropic phase with reduced packing fraction. Hence, the $I-N$ phase boundary shifts towards lower density for the cold particles and higher density for the hot particles. In Figs. 8(a) and 8(b) we see a continuous phase transition from a disordered to an ordered state for active systems, as is evident from the continuous increase in the nematic order parameter in contrast to the sudden jump in the order parameter for the equilibrium case. We notice a local minimum in $S_{\text {cold }}$ in the range $\eta=0.36-0.38$ [Fig. 8(a)]. To check the possible effects of the finite system size, we simulated a larger system with $N=4096$ SRSs and observed similar results (see Fig. 19 for details). The local minimum occurs due to the formation of multiple domains with different orientations of the nematic director, which effectively reduces the global

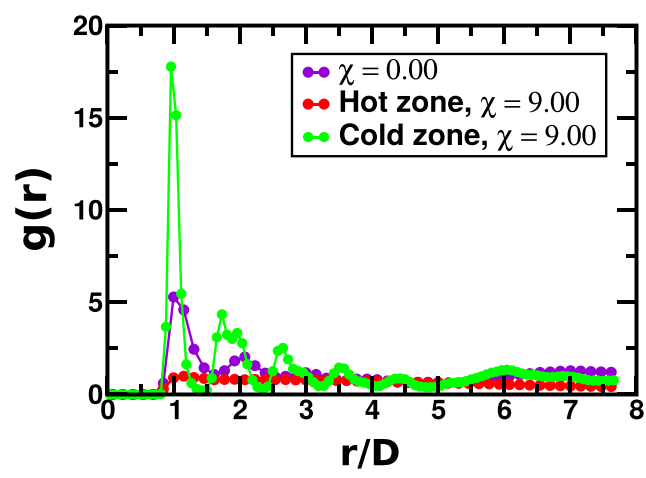

(a)

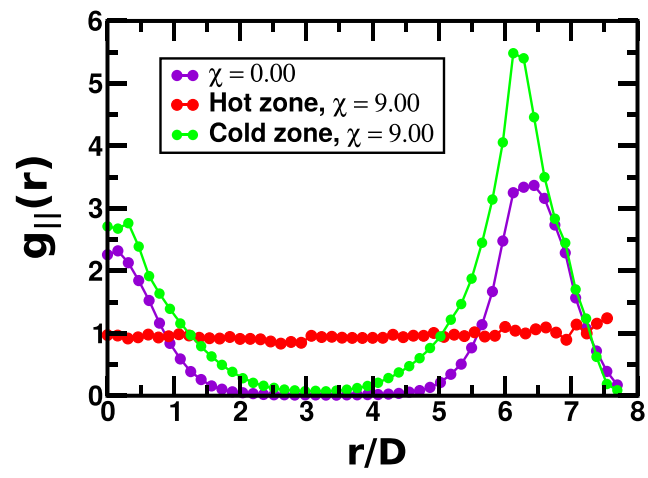

(c)

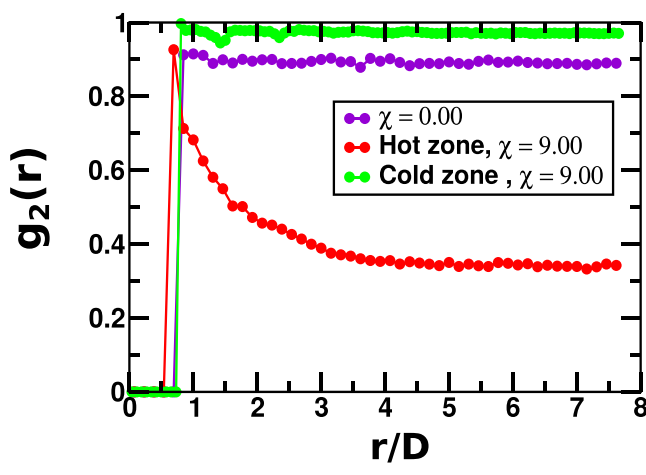

(b)

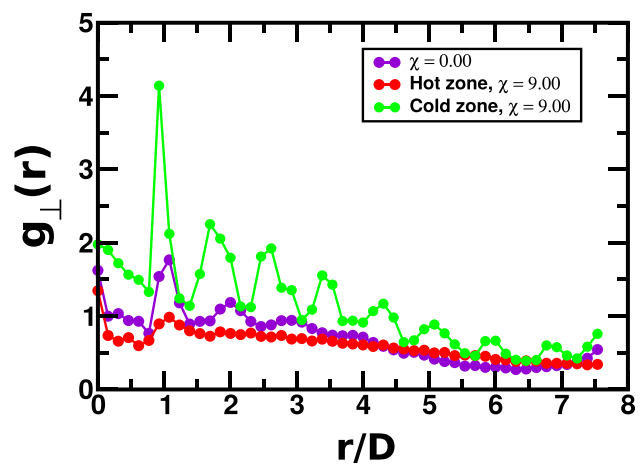

(d)

FIG. 12. Pair correlation functions in the segregated zones for a smectic initial phase $(\eta=0.67)$ at $\chi=9.00$ : (a) center-of-mass pair correlation function $g(r)$, (b) orientational pair correlation function $g_{2}(r)$, and projection of $g(r)$ for the distances $(\mathrm{c})$ parallel $\left[g_{\|}(r)\right]$ and (d) perpendicular $\left[g_{\perp}(r)\right]$ to the director of the spherocylinders. 


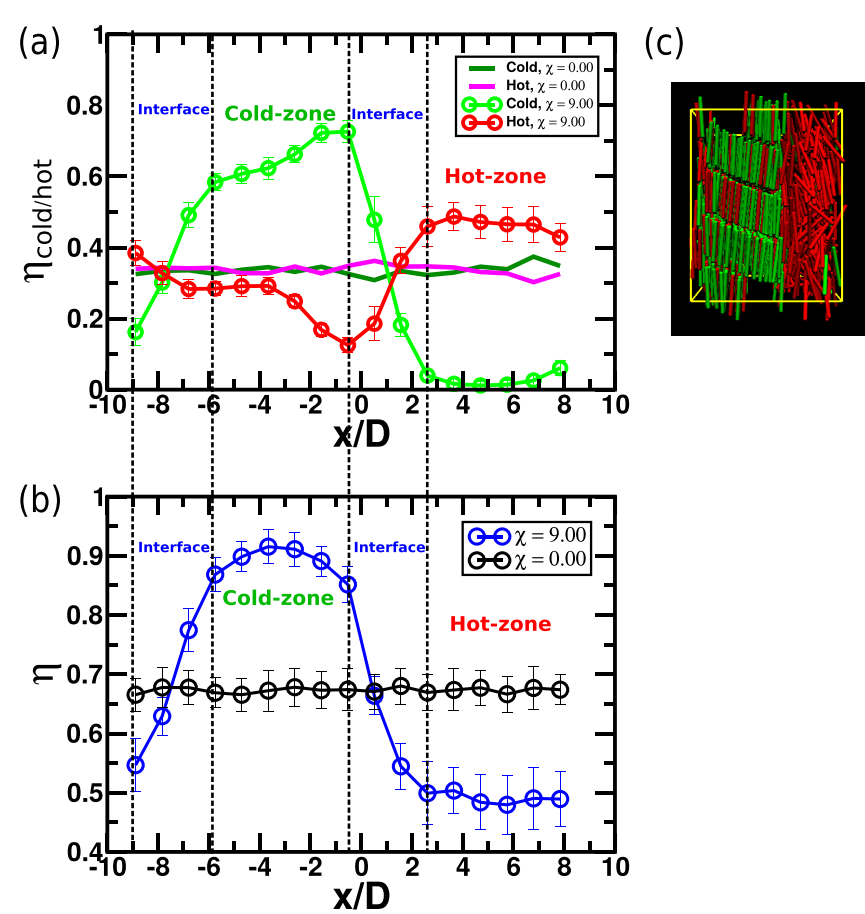

FIG. 13. (a) Packing fraction of cold $\eta_{\text {cold }}$ and hot $\eta_{\text {hot }}$ particles separately and (b) effective packing fraction of the particles present in each slab (including both hot and cold) in the direction perpendicular to the interface at $\eta=0.67$ and $\chi=9.00$. Location of the cold, hot, and interfacial regions are shown in the legend. The dotted lines indicate the boundary of each zone. (c) Snapshot of the system at steady state.

nematic order parameter of the cold particles. This is also verified by calculating orientational and positional pair correlation functions in the cold regions of the respective densities (Fig. 20). For other densities, we observe a single domain with a fixed orientation of the director in the cold zone which increases $S_{\text {cold }}$.

\section{Initial nematic configuration}

For the initial nematic configuration, phase separation starts at $\chi_{c}=1.50-2.00$. We find that activity drives the cold particles to undergo a nematic to smectic transition while the hot particles exhibit a nematic to isotropic transition as shown in Fig. 9. In Fig. 9(b) we can see that at $\chi=2.00$, the nematic order parameter in the cold zone is $S_{\text {cold }}=0.97$ and the packing fraction is $\eta_{\text {cold }}=0.73$. These values are consistent with the equilibrium smectic phase for SRSs with a shape anisotropy $L / D=5$ (Fig. 2). On the other hand, the hot particles develop isotropic structure with $S_{\text {hot }}=0.10$ and $\eta=0.38$. A further increase of $\chi$ turns the cold zone into a close-packed crystal structure as shown in Figs. 9(c) and 9(d).

\section{Initial smectic configuration}

In the case of an initial smectic configuration, the system starts to phase separate at very low activity: $\chi_{c}=0.61-1.00$. A small amount of temperature difference drives the cold zone into a close-packed crystal structure while the hot zone undergoes a transition to the nematic phase, as shown in Fig. 10. For $\chi=9.0$, the local nematic order parameters and packing fractions in the segregated regions are as follows: In the hot region, $S=0.58$ and $\eta=0.50$, which is consistent with the equilibrium nematic phase, and in the cold region, $S=0.98$ and $\eta=0.87$, which is consistent with the equilibrium crystal phase (Fig. 2). The observed phases are further verified by calculating suitable pair correlation functions as discussed in detail in Sec. III C.

\section{Initial crystal configuration}

In the case of an initial crystal configuration, we surprisingly find $\chi_{c}$ to be very high $\left(\chi_{c}=3.50-4.00\right)$ compared to the values at liquid phases. The reason is that many hot particles are stuck in the cold zone and these particles require a larger amount of energy to overcome the potential barrier for demixing. Another reason is that the relaxation in the crystal phase is very slow compared to that in liquid-crystal phases. Therefore, a longer time may be required to phase separate at smaller activity. After phase separation, the layered structure in the hot zone starts to break up into a nematiclike structure that appears to be a far-from-equilibrium nematic phase [Figs. 11(c) and 11(d)]. The local ordering is much higher compared to that in the usual nematic phase; however, the breakdown of the layered structure causes a significant decrease in the packing fraction: $S_{\text {hot }}=0.92$ and $\eta_{\text {hot }}=0.62$ at $\chi=9.00$. Another important point to note is that, while melting, the hot particles do not go through a smectic phase. This differentiates the melting transition in the active subsystem from the equilibrium one.

\section{Pair correlation functions}

The local ordering in segregated zones is further characterized by calculating relevant pair correlation functions $[69,70]$. Apart from the radial distribution function $g(r)$, we also calculate the orientational pair correlation function $g_{2}(r)$, which is relevant for quantifying nematic order. Here $g_{2}(r)$ is defined as the second-order Legendre polynomial associated with the orientation vectors $\vec{u}_{i}$ and $\vec{u}_{j}$ of two spherocylinders $i$ and $j$ separated by distance $r: g_{2}(r)=\left\langle P_{2}\left(\vec{u}_{i} \cdot \vec{u}_{j}\right)\right\rangle$. We further calculate the vectorial pair correlation functions $g_{\|}(r)$ and $g_{\perp}(r)$, which are the projections of the radial distribution function $g(r)$ in the directions parallel and perpendicular to the nematic director, respectively. Periodic oscillations in $g_{\|}(r)$ indicate the presence of layering and thus differentiate between nematic and smectic phases. The $g_{\perp}(r)$ indicates the presence of in-layer periodicity and thus differentiates between smectic and crystal phases.

In Fig. 12 we plot the pair correlation functions for a system starting from a smectic phase at $\eta=0.67$ and $\chi=9.00$. The correlation functions are calculated in the hot and cold zones separately over a sphere of diameter $16 D$ to quantify both short- and long-range correlations. As shown in Sec. III B 3, this system shows crystalline structure $(S=0.99)$ in the cold zone and nematic structure in the hot zone $(S=$ $0.63)$ at this activity. From the pair correlation function $g(r)$ shown in Fig. 12(a), we observe a significant increase of the height of the first and second peaks in the cold zone compared to the nonactive case and the emergence of a third peak. This is a signature of high positional correlation among the cold particles. In contrast, in the hot zone, we observe 


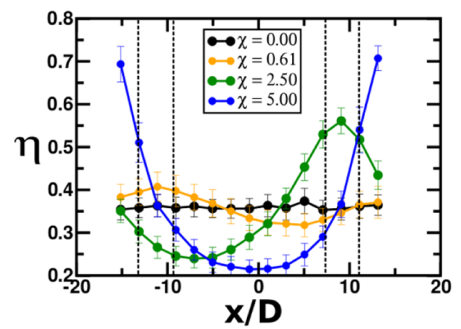

(a)

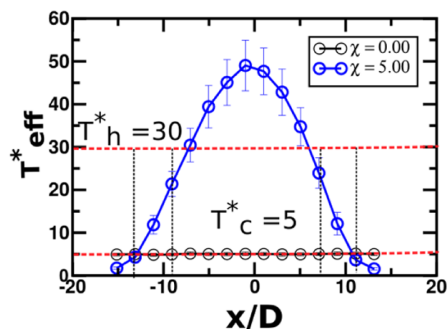

(b)

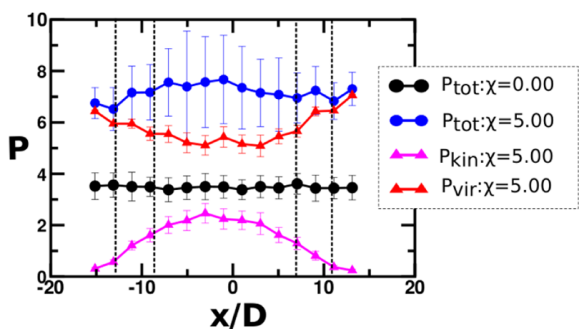

(c)

FIG. 14. (a) Effective packing fraction $\eta$, (b) temperature $T_{\text {eff }}^{*}$, and (c) pressure $P$ profile in the direction perpendicular to the interface for different activities $\chi$ at $\eta=0.36$. The region between black vertical dashed lines indicates the location of the interface for $\chi=5$. Red horizontal dashed lines in (b) indicate the temperatures imposed on hot and cold particles at $\chi=5.00$. In (c) we decompose the total pressure of the active system into kinetic and virial parts. It is clearly visible that the kinetic pressure is decreasing and the virial pressure is increasing from the hot to the cold zone. The 0 is the position of the slab located at half of the box length in the direction perpendicular to the interfacial plane.

that the height of the first peak decreases significantly and the second peak vanishes. However, orientational correlations [Fig. 12(b)] still exist, which identify the phase as nematic. Periodic oscillations in $g_{\|}(r)$ indicate the presence of a layered structure in the cold zone. The distance between two successive peaks is around 6.00, which is the end to end distance of a spherocylinder $(L / D+1)$. Multiple peaks at equal distance in $g_{\perp}(r)$ signify high translational ordering within the layer, which confirms the emergence of local crystalline structure in the cold particles' cluster.

\section{Interfacial properties}

To obtain interfacial properties, we divide the simulation box into a number of slabs $N_{\text {slabs }}$. Here $N_{\text {slabs }}$ is chosen such that each slab contains enough particles (in our case, about 50) to get stable statistics. The effective density and temperature of the $i$ th slab are calculated as

$$
\begin{gathered}
\eta(i)=\frac{n(i)}{v(i)} v_{\mathrm{hsc}}, \\
5 \times \frac{1}{2} k_{B} T_{\mathrm{eff}}(i)=\frac{1}{n(i)} \sum_{j=1}^{n(i)}\left(\frac{1}{2} m v_{j}^{2}+\frac{1}{2} I \omega_{j}^{2}\right) .
\end{gathered}
$$

Here $n(i)$ and $v(i)$ are the number of particles and volume of the $i$ th slab, respectively; $v_{j}$ and $\omega_{j}$ indicate the translational and rotational velocities of the SRS $j$, respectively. In Eq. (6), the term 5 appears on the left-hand side as the total number of degrees of freedom for a rigid spherocylinder is 5, arising from 3 translational and 2 rotational motions. We identify the locations of the phase-separated zones and the interface by calculating the local packing fractions of hot $\left(\eta_{\text {hot }}\right)$ and cold particles $\left(\eta_{\text {cold }}\right)$ as shown in Fig. 13(a). We observe that the effective packing fraction of each slab $\eta$ (including both hot and cold particles) decreases in the hot zone and increases in the cold zone compared to the initial equilibrium system [Fig. 13(b)]. The region where $\eta$ changes sharply from one zone to another is defined as the interface. Analyzing the values of $\psi$ for each subbox as defined in Sec. III A and the snapshots of the phase-separated systems shown in Figs. 3 and 7,9-11 indicate that the interface preferentially lies in a plane parallel to one of the sides of the simulation box. Our system exhibits two interfaces due to the effect of the periodic boundary condition. The interface occupies a finite region of the simulation box and the width of the interface does not show any significant dependence on the system size, as shown in Fig. 21. In Fig. 14(a) we plot the effective $\eta$ at

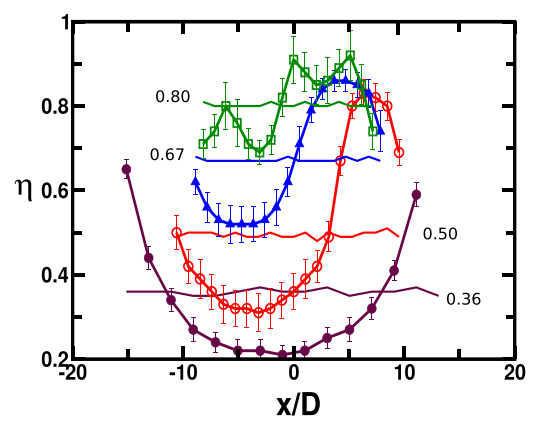

(a)

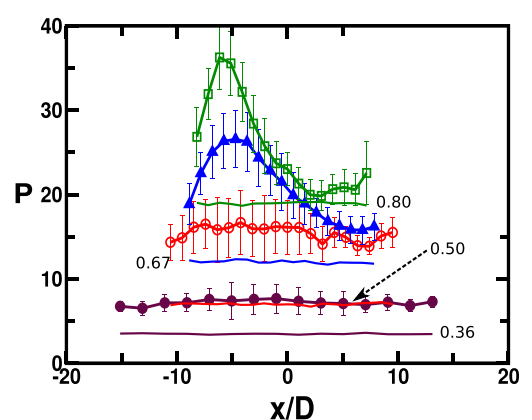

(b)

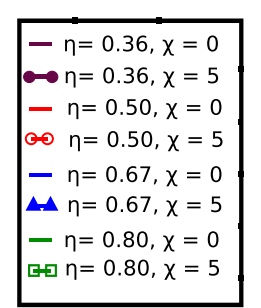

FIG. 15. (a) Effective packing fraction $\eta$ and (b) pressure $P$ profile in the direction perpendicular to the interface for $\chi=0.00$ and 5.00 at different densities corresponding to the different initial phases: $\eta=0.36$ for the initial isotropic phase, $\eta=0.50$ for the initial nematic phase, $\eta=0.67$ for the initial smectic phase, and $\eta=0.80$ for the initial crystal phase. The pressure is roughly constant within error bars for lower-density phases $(I$ and $N$ ) but it decreases continuously from the hot to the cold zone for higher-density phases $(\operatorname{Sm} A$ and $K$ ). The equilibrium packing fraction corresponding to each solid line is labeled. The 0 is the position of the slab located at half of the box length in the direction perpendicular to the interfacial plane. 


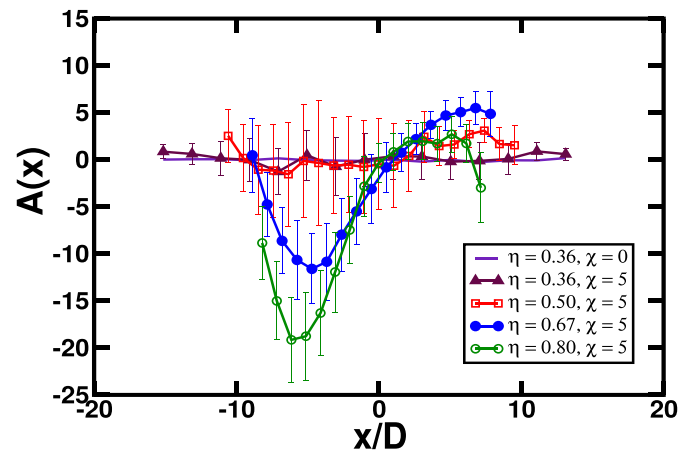

(a)

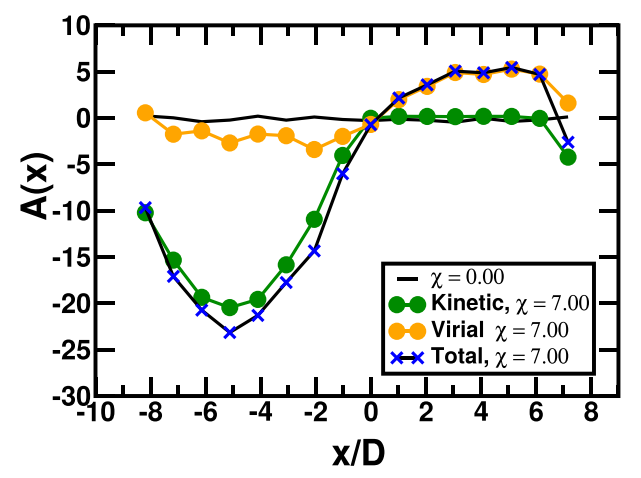

(b)

FIG. 16. (a) Pressure anisotropy $A$ as a function of distance $x$ in the direction perpendicular to the interface for different packing fractions at $\chi=0.00,5.00$. (b) Plot of $A(x)$ in the initial crystal phase $(\eta=0.80)$ for $\chi=7.00$. Here $A(x)$ is decomposed into kinetic and virial parts. The pressure anisotropy is dominated by the kinetic part in the hot zone and the virial part in the cold zone. The 0 is the position of the slab located at half of the box length in the direction perpendicular to the interfacial plane.

different activities and find that the spatial inhomogeneity in $\eta$ increases with the increase of activity.
The effective temperature decreases continuously from the hot to the cold zone [Fig. 14(b)]. The coexistence of two

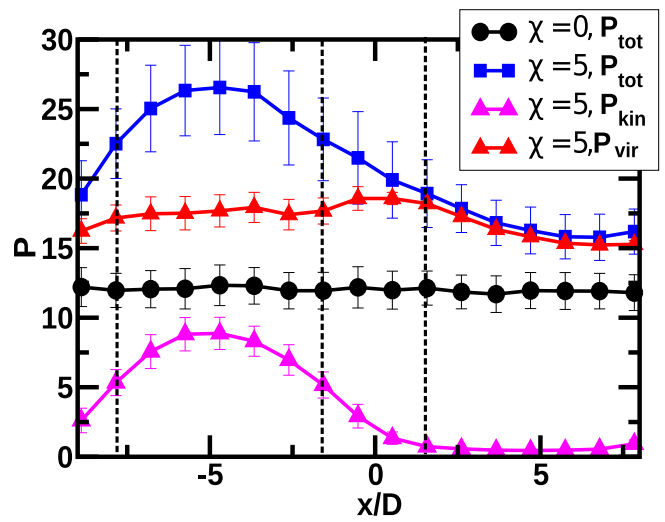

(a)

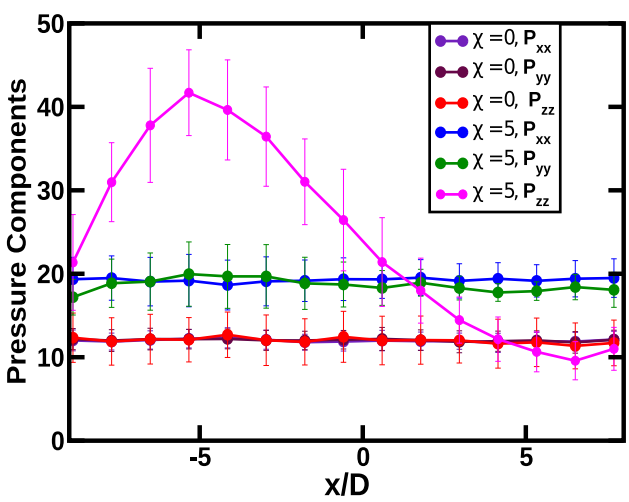

(b)

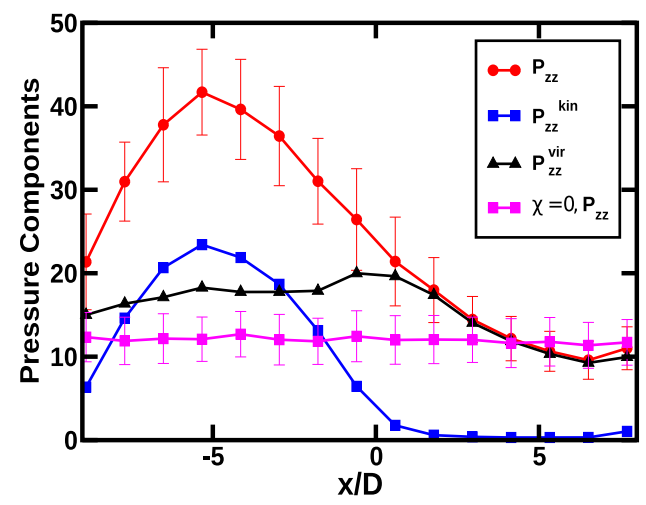

(c)

FIG. 17. (a) Pressure profile for $\eta=0.67$ at $\chi=0.00,5.00$ in the direction perpendicular to the interface. Here the total pressure decreases continuously from the hot to the cold zone. The anisotropy in pressure is dominated by the kinetic contribution in the hot zone and the virial contribution (arising from the WCA interaction) in the cold zone. The region between the dotted lines indicates the interface. (b) Spatial variation of pressure components across the interface. Here $P_{x x}$ is the normal component and $P_{y y}$ and $P_{z z}$ are the tangential components. The anisotropy of pressure is coming from the $P_{z z}$ component, which is also the nematic director of the hot zone. (c) Here $P_{z z}$ is decomposed into kinetic and virial parts. 
temperatures at the hot-cold interface signifies the nonequilibrium nature of the system. However, it is surprising that in the steady state the cold zone has regions where the local temperature is lower than the imposed cold particles' temperature $T_{c}^{*}$ and in some regions of the hot zone the local temperature is much higher than the imposed hot particles' temperature $T_{h}^{*}$. In Fig. 14(b) we see that the maximum temperature in the hot zone is around 50, which is much higher than $T_{h}^{*}=30$. In the cold zone, the minimum temperature is around 2 , which is lower than $T_{c}^{*}=5$. However, the effective temperature averaged over all the hot particles $T_{h}^{\text {eff }}$ is lower than $T_{h}^{*}$ and the effective temperature averaged over all the cold particles $T_{c}^{\text {eff }}$ is higher than $T_{c}^{*}$ due to heat exchange between them as mentioned in Eq. (2).

We evaluate the pressure profile from diagonal components of the stress tensor

$$
\begin{gathered}
P_{\mathrm{kin}}(i)=\frac{1}{3 \times V(i)} \sum_{j=1}^{n(i)} m v_{j}^{2}, \\
P_{\mathrm{vir}}(i)=\frac{1}{3 \times V(i)} \sum_{j=1}^{n(i)-1} \sum_{k>j} \vec{r}_{j k} \cdot \vec{f}_{j k}, \\
P(i)=\left\langle P_{\mathrm{kin}}(i)+P_{\mathrm{vir}}(i)\right\rangle_{s s} .
\end{gathered}
$$

Here $P, P_{\text {kin }}$, and $P_{\text {vir }}$ designate total, kinetic, and virial pressures, respectively; $P_{\text {vir }}$ arises due to the particles' interaction, which is defined as the product of the relative distance $\vec{r}_{j k}$ and interacting force $\vec{f}_{j k}$ between the SRSs $j$ and $k$. We observe that the local pressure increases with activity and it is nearly constant within error bars across the hot-cold interface [Fig. 14(c)]. This is due to the fact that higher temperature causes higher kinetic pressure in the hot zone that acts at the hot-cold interface, inducing higher packing and ordering in the cold zone. This enhances the virial pressure in the cold zone, resulting in the total pressure being constant across the interface. This is shown in Fig. 14(c), where we decompose the total pressure into kinetic and virial parts for the system with $\eta=0.36$ and $\chi=5.00$. However, this behavior is found only for the lower densities ( $I$ and $N$ initial phases). In the case of higher densities (Sm and $K$ initial phases), we observe that the total pressure decreases continuously from the hot to the cold zone (Fig. 15). This can be rationalized as follows: In the case of smectic and crystal initial phases, along with the kinetic pressure, the virial pressure is also high in the hot zone due to their high orientational order [as the hot zone shows nematiclike structure (Figs. 10 and 11)]. As a result, the total pressure increases in the hot zone, which cannot be compensated in the cold zone by increasing the virial pressure only. We calculate the pressure anisotropy $A(x)$, which is defined as

$$
A(x)=P_{n}(x)-P_{t}(x) .
$$

Here $P_{n}(x)$ and $P_{t}(x)$ are the normal and tangential components of the total pressure, respectively, in the directions perpendicular and parallel to the interface. We designate the perpendicular direction of the hot-cold interface as $x$ and the other two directions parallel to the interfacial plane as $y$ and $z$. Thus the pressure components are defined as $P_{n}(x)=P_{x x}(x)$ and $P_{t}(x)=\left[P_{y y}(x)+P_{z z}(x)\right] / 2$. In equilibrium, the pressure is isotropic and therefore $A(x)=0$. In the active system, we observe that the anisotropy is close to zero within the error bars for lower densities ( $I$ and $N$ initial phases). However, it increases for higher densities (Sm and $K$ initial phases) as shown in Fig. 16(a). To understand this precisely, we plot different pressure components across the interface as shown in Fig. 17 and find that the anisotropy in higher densities may arise due to the effect of the active stress of the hot particles along their nematic director, which acts parallel to the interfacial plane. To understand if the anisotropy in the pressure tensor is a consequence of the constant-volume simulation $(N V T)$, we perform a constant-pressure simulation (NPT) with an orthorhombic boundary condition and do not find any significant change in the pressure anisotropy. In Refs. [71-73] it was reported that for the equilibrium NPT simulation, the diagonal components of the pressure tensor become unequal in spatially ordered phases (smectic and crystal) due to maintaining a constant cubic shape throughout the simulation. In our case, the NPT simulation is done in orthorhombic boundary conditions; hence this issue is not applicable in our case.

To understand possible system-size effects, we perform a similar analysis with $N=4096$ particles, keeping all other system parameters and simulation protocol unchanged; we do not find any significant system size effect. The different results for two system sizes are compared in Figs. 19, 20, and 21.

\section{CONCLUSION AND OUTLOOK}

In summary, we have presented a simple two-temperature model to study the thermodynamic and structural properties of active-passive spherocylinders where the level of activity is modeled by maintaining a temperature difference between the hot and cold particles. Starting from different initial equilibrium phases $(I, N$, and $\mathrm{Sm})$, we showed that our simple model leads to not only phase separation into hot and cold regions but also liquid-crystal ordering of the cold particles and opposite shifts of the phase boundaries for mesophase formation, with respect to the equilibrium case, in the cold and hot domains. The extent of phase separation was quantified by an order parameter based on the local density. We found that the critical activity for phase separation lies in a small range $1<\chi_{c}<4$ for a wide range of densities from the isotropic to the crystal phase. This interesting observation highlights the two-temperature model as an experimentally feasible system for studying the effect of scalar activity in colloidal rods. We observed that the critical activity decreases with density in the liquid regime and increases again in the crystal regime. Based on these observations, a phase diagram was drawn in the state phase $\chi$ vs $\eta$, showing the parametric regions of phase-separated and homogeneously mixed states.

We found the segregated zones developing different liquidcrystal structures depending on the activity and initial phase of the system. For example, an initial isotropic configuration shows nematic ordering in the cold region, which eventually turns into crystalline ordering at higher activities. Similarly, an initial nematic configuration shows smectic or crystal ordering in the cold zone, depending on the value of $\chi$, and isotropic structure in the hot zone. As a result, the $I-N$ phase boundary shifts towards higher density for the hot particles and lower density for the cold particles. The segregated structures are identified by calculating the local nematic order parameter and different pair correlation functions. Finally, we analyzed 


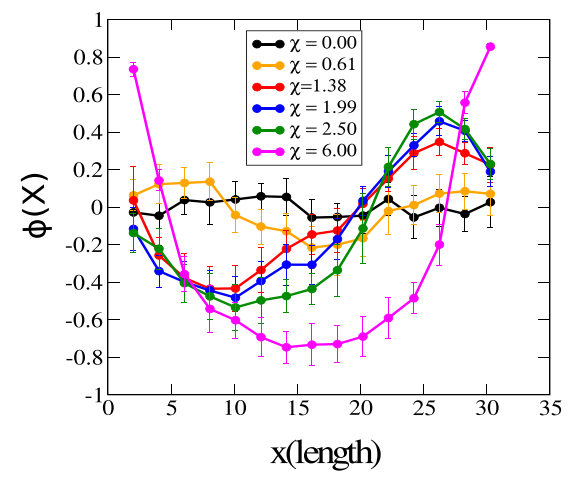

(a)

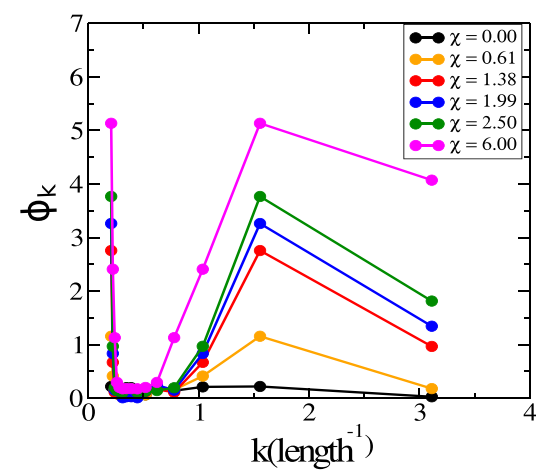

(b)

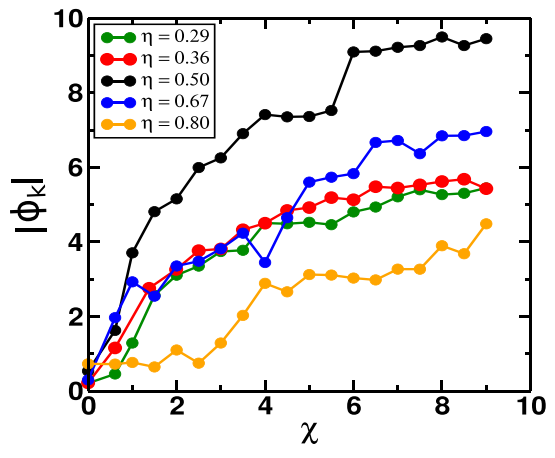

(c)

FIG. 18. (a) Density order parameter $\phi$ at $\eta=0.36$ for different activities $\chi$ in the direction normal to the interface. (b) Fourier components of $\phi(x)$. Here $k=2 \pi / x$. In this figure we can see two peaks in $\phi_{k}$ : The peak at the lowest $k$ value (around $k=0.2 \Rightarrow x=31.42$, which is approximately the length of the box $L$ ) indicates the occurrence of phase separation on the macroscopic level and the other peak (around $k=1.5 \Rightarrow x=4.2$ ) indicates microscopic phase separation. The amount of phase separation is determined from the height of the first peak in $\phi_{k}$. (c) Magnitude of the Fourier component of $\phi$ for the smallest $k$ vs activity $\chi$ at several packing fractions $\eta$. The trend shown is similar to that in Fig. 4.

interfacial profiles of various thermodynamic quantities and concluded that the order-disorder transitions in the segregated zones are probably governed by a local balance of pressure across the interface: Higher temperature induces higher kinetic pressure in the hot zone, which is compensated in the cold zone by an increased virial pressure.

Another possible reason for the order-disorder transition may be an entropic effect. The hot particles compensate for the loss of entropy due to the ordering transition in the cold zone by developing a disordered structure. An important component of future work will be to examine configurational entropy and free energy, as well as entropy production and currents, to shed light on the mechanisms underlying the nonequilibrium phase transitions we observe. Finally, analytical theories of two-temperature models so far have been limited to spherical particles [27-29]. Hence, generalizing their theoretical approach to make analytical predictions for two-temperature models with anisotropic particles is an important challenge.

\section{ACKNOWLEDGMENTS}

We would like to thank Prof. Aparna Baskaran and Prof. Yves Lansac for helpful discussions. We thank DAE, India for financial support by providing the computational facility. J.C. acknowledges support through an INSPIRE fellowship. S.R. was supported by a J C Bose Fellowship of the SERB, India, and by the Tata Education and Development Trust, and acknowledges discussions during the KITP program on Symmetry, Thermodynamics, and Topology in Active Matter. This research was supported in part by the National Science Foundation under Grant No. NSF PHY-1748958. C.D. was supported by a Distinguished Fellowship of the SERB, India.

\section{APPENDIX: QUANTIFYING MACROSCOPIC PHASE SEPARATION}

To quantify if the phase-separation happens in the macroscopic level, we have used the following criterion: We divide

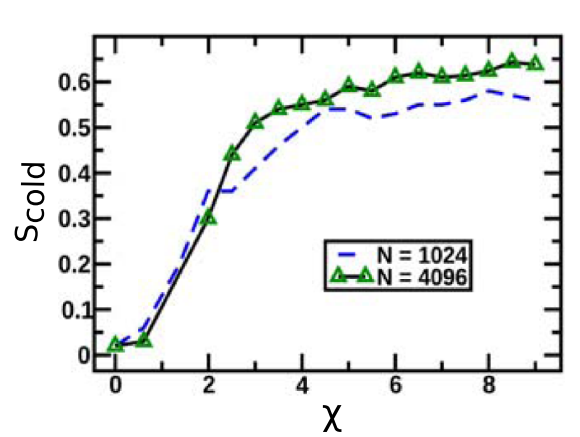

(a)

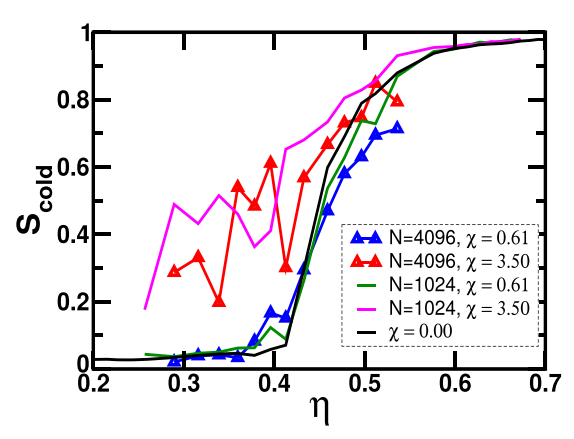

(b)

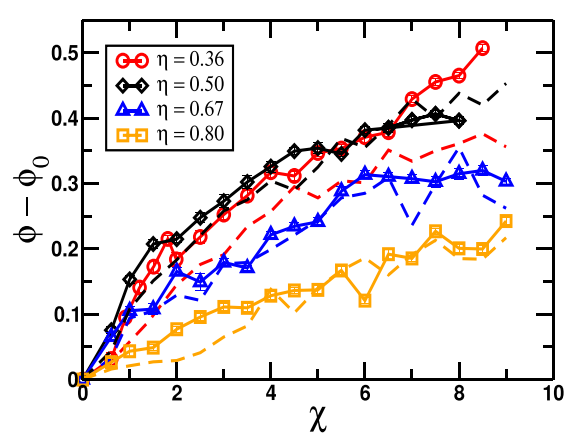

(c)

FIG. 19. (a) Average nematic order parameter of cold particles $S_{\text {cold }}$ with activity $\chi$ for $N=4096$ (solid line) compared to the earlier case with $N=1024$ (dashed line) at $\eta=0.36$. (b) Plot of $S_{\text {cold }}$ vs $\eta$ at different $\chi$ as given in the legend. Here we also observe local minima for a certain range of $\eta$ as it is observed in the earlier case with $N=1024$ [Fig. 8(a)]. (c) Density order parameter $\phi$ with $\chi$ for the larger system size with $N=4096$ at different packing fractions. The density order parameters in the system with $N=1024$ particles are designated by dotted lines at the respective packing fractions. We do not observe any significant system size effect for these cases. 


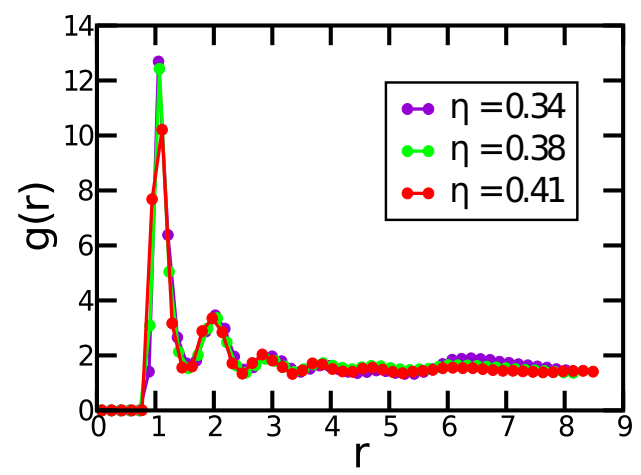

(a)

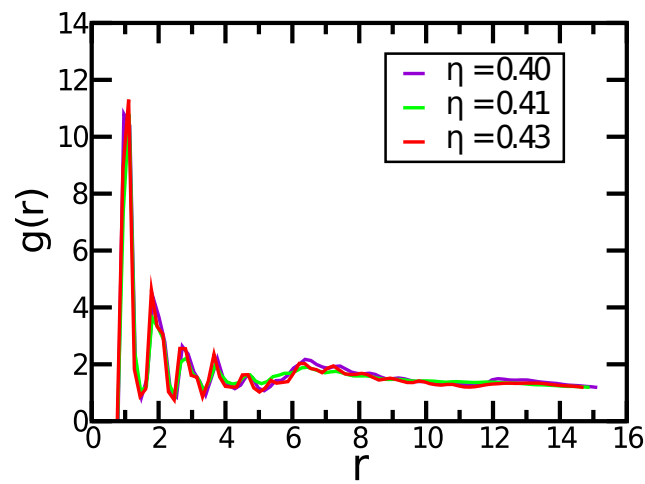

(c)

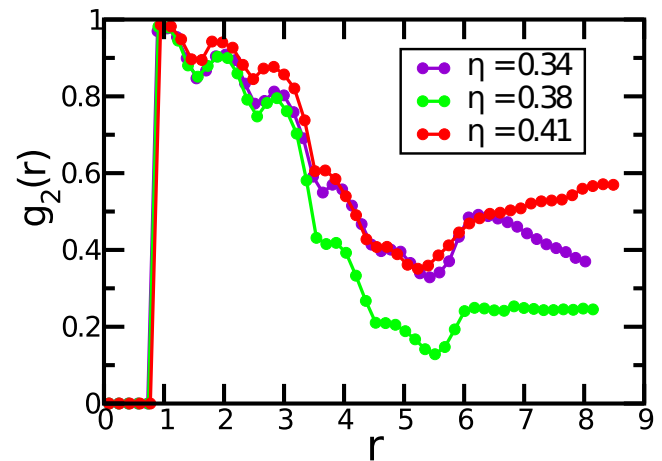

(b)

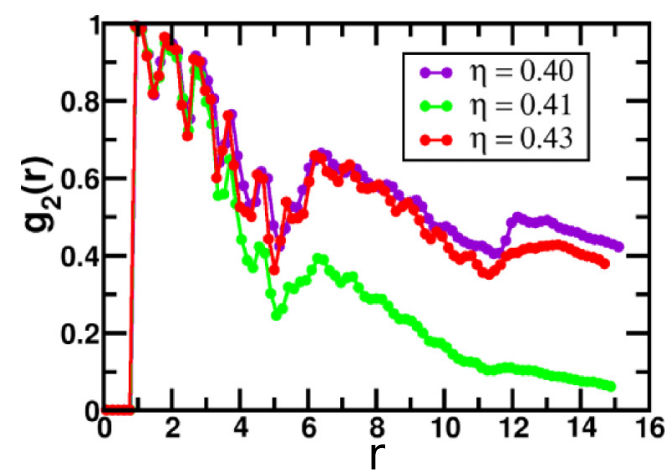

(d)

FIG. 20. Pair correlation functions for $\chi=3.50$ at the values of $\eta$ around which we find local minima in $S_{\text {cold }}$ for $N=1024$ and 4096 as mentioned in Fig. 19(b). The local minima occur at values of $\eta=0.38$ and 0.41 for $N=1024$ and 4096, respectively. For both cases, the orientational correlation $g_{2}(r)$ decreases and saturates at a smaller value at large distances [(b) and (d)]. This is due to the presence of local domains of different average orientations of the nematic director, which effectively decreases the global ordering of the cold particles. For other packing fractions, $g_{2}(r)$ saturates at a higher value at large distances. Here we observe a stable single domain of a fixed orientation of the nematic director in the cold zone. The translational correlation $g(r)$ [(a) and (c)] is nearly independent of $\eta$ in the range considered.

the simulation box into a number of slabs $N_{\text {slabs }}$ in the direction normal to the interface. Here $N_{\text {slabs }}$ is chosen such that each slab contains enough particles (in our case, about 50) to get stable statistics. For each slab $i$, we calculate the number difference of hot $\left(n_{h}^{i}\right)$ and cold $\left(n_{c}^{i}\right)$ particles divided

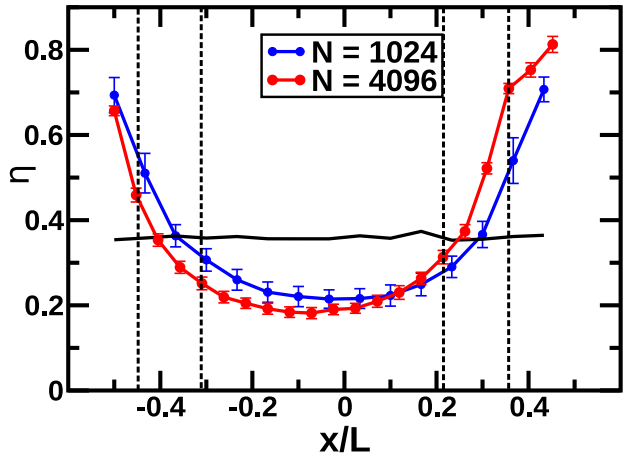

(a) by the total number of particles $n_{\text {tot }}^{i}$ in that slab. We then define

$$
\phi_{i}=\left\langle\frac{n_{c}^{i}-n_{h}^{i}}{n_{c}^{i}+n_{h}^{i}}\right\rangle_{s s}
$$

FIG. 21. (a) Effective packing fraction $\eta$ and (b) temperature $T_{\text {eff }}^{*}$ profile in the direction perpendicular to the interface scaled by the box size for $N=4096$ and 1024 at $\eta=0.36$ and $\chi=5.00$. The black horizontal solid line in (a) indicates the local packing fraction in the absence of activity. Black vertical dotted lines in (a) and (b) indicate the interfacial region. Red horizontal dashed lines in (b) indicate the temperatures imposed on hot and cold particles. For both system sizes, the width of the interfacial region is about 5 in units of the diameter $D$ of the SRS. 
where $\langle\cdots\rangle_{\text {ss }}$ represents a steady-state average over a sufficiently large number of configurations. The average of $\phi_{i}$ over all slabs will be 0 as $\phi_{i}$ varies symmetrically from -1 to +1 from the hot-rich to the cold-rich zone. We therefore calculate the Fourier transformation of $\phi(x)$, where $x$ measures the position of the slab. The magnitude of the first nonvanishing Fourier component $\left|\phi_{k}\right|$ is the measure of macroscopic phase separation in our system (Fig. 18). In Fig. 18(b) the first peak occurs at $x=31.42$, which is approximately the length of our simulation box $(L=32)$. This indicates the occurrence of phase separation in a macroscopic scale. In Fig. 18(c) we plot the magnitude of $\left|\phi_{k}\right|$ for the smallest $k$ as a function of the activity at several packing fractions, which shows a trend similar to that in Fig. 4.
[1] M. C. Marchetti, J. F. Joanny, S. Ramaswamy, T. B. Liverpool, J. Prost, M. Rao, and R. A. Simha, Rev. Mod. Phys. 85, 1143 (2013).

[2] S. Ramaswamy, Annu. Rev. Condens. Matter Phys. 1, 323 (2010).

[3] P. Romanczuk, M. Bär, W. Ebeling, B. Lindner, and L. Schimansky-Geier, Eur. Phys. J.: Spec. Top. 202, 1 (2012).

[4] M. E. Cates and J. Tailleur, Annu. Rev. Condens. Matter Phys. 6, 219 (2015).

[5] S. Ramaswamy, R. A. Simha, and J. Toner, Europhys. Lett. 62, 196 (2003).

[6] J. Toner, Y. Tu, and S. Ramaswamy, Ann. Phys. (NY) 318, 170 (2005).

[7] J. Toner and Y. Tu, Phys. Rev. Lett. 75, 4326 (1995).

[8] J. Toner and Y. Tu, Phys. Rev. E 58, 4828 (1998).

[9] I. Buttinoni, J. Bialké, F. Kümmel, H. Löwen, C. Bechinger, and T. Speck, Phys. Rev. Lett. 110, 238301 (2013).

[10] J. Bialké, J. T. Siebert, H. Löwen, and T. Speck, Phys. Rev. Lett. 115, 098301 (2015).

[11] C. Bechinger, R. Di Leonardo, H. Löwen, C. Reichhardt, G. Volpe, and G. Volpe, Rev. Mod. Phys. 88, 045006 (2016).

[12] S. Mandal, B. Liebchen, and H. Löwen, Phys. Rev. Lett. 123, 228001 (2019).

[13] H. Löwen, J. Chem. Phys. 152, 040901 (2020).

[14] H. R. Vutukuri, Z. Preisler, T. H. Besseling, A. van Blaaderen, M. Dijkstra, and W. T. Huck, Soft Matter 12, 9657 (2016).

[15] G. S. Redner, M. F. Hagan, and A. Baskaran, Phys. Rev. Lett. 110, 055701 (2013).

[16] T. Speck, J. Bialké, A. M. Menzel, and H. Löwen, Phys. Rev. Lett. 112, 218304 (2014).

[17] S. Saha, J. Agudo-Canalejo, and R. Golestanian, Phys. Rev. X 10, 041009 (2020).

[18] E. Tjhung, C. Nardini, and M. E. Cates, Phys. Rev. X 8, 031080 (2018).

[19] J. Stenhammar, R. Wittkowski, D. Marenduzzo, and M. E. Cates, Phys. Rev. Lett. 114, 018301 (2015).

[20] R. R. Netz, Phys. Rev. E 101, 022120 (2020).

[21] S. Chaki and R. Chakrabarti, Physica A 511, 302 (2018).

[22] S. Chaki and R. Chakrabarti, J. Chem. Phys. 150, 094902 (2019).

[23] S. R. McCandlish, A. Baskaran, and M. F. Hagan, Soft Matter 8, 2527 (2012).

[24] M. R. Shaebani, A. Wysocki, R. G. Winkler, G. Gompper, and H. Rieger, Nat. Rev. Phys. 2, 181 (2020).

[25] S. K. Das, J. Chem. Phys. 146, 044902 (2017).

[26] N. Ganai, S. Sengupta, and G. I. Menon, Nucleic Acids Res. 42, 4145 (2014).

[27] A. Y. Grosberg and J.-F. Joanny, Phys. Rev. E 92, 032118 (2015).
[28] A. Y. Grosberg and J.-F. Joanny, Polymer Sci. C 60, 118 (2018).

[29] E. Ilker and J.-F. Joanny, Phys. Rev. Res. 2, 023200 (2020).

[30] S. N. Weber, C. A. Weber, and E. Frey, Phys. Rev. Lett. 116, 058301 (2016).

[31] J. Smrek and K. Kremer, Phys. Rev. Lett. 118, 098002 (2017).

[32] J. Smrek and K. Kremer, Entropy 20, 520 (2018).

[33] S. S. N. Chari, C. Dasgupta, and P. K. Maiti, Soft Matter 15, 7275 (2019).

[34] J. Bartnick, A. Kaiser, H. Löwen, and A. V. Ivlev, J. Chem. Phys. 144, 224901 (2016).

[35] A. V. Ivlev, J. Bartnick, M. Heinen, C.-R. Du, V. Nosenko, and H. Löwen, Phys. Rev. X 5, 011035 (2015).

[36] R. Soto and R. Golestanian, Phys. Rev. Lett. 112, 068301 (2014).

[37] V. Schaller, C. Weber, C. Semmrich, E. Frey, and A. R. Bausch, Nature (London) 467, 73 (2010).

[38] Y. Sumino, K. H. Nagai, Y. Shitaka, D. Tanaka, K. Yoshikawa, H. Chaté, and K. Oiwa, Nature (London) 483, 448 (2012).

[39] P.-G. de Gennes and J. Prost, The Physics of Liquid Crystals (Oxford University Press, Oxford, 1993), Vol. 83.

[40] T. Vicsek and A. Zafeiris, Phys. Rep. 517, 71 (2012).

[41] H. Chaté, F. Ginelli, and R. Montagne, Phys. Rev. Lett. 96, 180602 (2006).

[42] S. Mishra and S. Ramaswamy, Phys. Rev. Lett. 97, 090602 (2006).

[43] F. Peruani, A. Deutsch, and M. Bär, Phys. Rev. E 74, 030904(R) (2006).

[44] S. Weitz, A. Deutsch, and F. Peruani, Phys. Rev. E 92, 012322 (2015).

[45] F. Ginelli, F. Peruani, M. Bär, and H. Chaté, Phys. Rev. Lett. 104, 184502 (2010).

[46] L. Huber, R. Suzuki, T. Krüger, E. Frey, and A. Bausch, Science 361, 255 (2018).

[47] Y. Yang, V. Marceau, and G. Gompper, Phys. Rev. E 82, 031904 (2010).

[48] M. C. Bott, F. Winterhalter, M. Marechal, A. Sharma, J. M. Brader, and R. Wittmann, Phys. Rev. E 98, 012601 (2018).

[49] R. A. Simha and S. Ramaswamy, Phys. Rev. Lett. 89, 058101 (2002).

[50] Y. Hatwalne, S. Ramaswamy, M. Rao, and R. A. Simha, Phys. Rev. Lett. 92, 118101 (2004).

[51] S. P. Thampi, A. Doostmohammadi, R. Golestanian, and J. M. Yeomans, Europhys. Lett. 112, 28004 (2015).

[52] S. Santhosh, M. R. Nejad, A. Doostmohammadi, J. M. Yeomans, and S. P. Thampi, J. Stat. Phys. 180, 699 (2020).

[53] A. Maitra and R. Voituriez, Phys. Rev. Lett. 124, 048003 (2020).

[54] J. D. Weeks, D. Chandler, and H. C. Andersen, J. Chem. Phys. 54, 5237 (1971). 
[55] M. S. Al-Barwani and M. P. Allen, Phys. Rev. E 62, 6706 (2000).

[56] M. P. Allen, G. T. Evans, D. Frenkel, and B. Mulder, Adv. Chem. Phys. 86, 1 (1993).

[57] C. Vega and S. Lago, Comput. Chem. 18, 55 (1994).

[58] D. J. Earl, J. Ilnytskyi, and M. R. Wilson, Mol. Phys. 99, 1719 (2001).

[59] D. M. Heyes, P. Turner, R. J. English, R. Williams, and A. C. Brańka, Phys. Rev. E 91, 042134 (2015).

[60] L. Verlet, Phys. Rev. 159, 98 (1967).

[61] I. P. Omelyan, Comput. Phys. 12, 97 (1998).

[62] N. S. Martys and R. D. Mountain, Phys. Rev. E 59, 3733 (1999).

[63] M. Rotunno, T. Bellini, Y. Lansac, and M. A. Glaser, J. Chem. Phys. 121, 5541 (2004).

[64] P. K. Maiti, Y. Lansac, M. A. Glaser, and N. A. Clark, Phys. Rev. Lett. 88, 065504 (2002).
[65] Y. Lansac, P. K. Maiti, N. A. Clark, and M. A. Glaser, Phys. Rev. E 67, 011703 (2003).

[66] H. J. C. Berendsen, J. P. M. Postma, W. F. van Gunsteren, A. DiNola, and J. R. Haak, J. Chem. Phys. 81, 3684 (1984).

[67] A. Cuetos, B. Martínez-Haya, L. Rull, and S. Lago, J. Chem. Phys. 117, 2934 (2002).

[68] A. Cuetos and B. Martínez-Haya, Mol. Phys. 113, 1137 (2015).

[69] S. C. McGrother, D. C. Williamson, and G. Jackson, J. Chem. Phys. 104, 6755 (1996).

[70] P. Bolhuis and D. Frenkel, J. Chem. Phys. 106, 666 (1997).

[71] H. Dominguez, E. Velasco, and J. Alejandre, Mol. Phys. 100, 2739 (2002).

[72] M. Bates and G. Luckhurst, J. Chem. Phys. 110, 7087 (1999).

[73] R. Hashim, G. Luckhurst, and S. Romano, J. Chem. Soc. Faraday Trans. 91, 2141 (1995). 